\title{
Fecal Microbial Transplant- A New Hope of Treatment for Ulcerative Colitis
}

\author{
Hena Butta ${ }^{1 *}$, Akshay Kapoor ${ }^{2}$, Anupam Sibal ${ }^{2}$, Raman Sardana ${ }^{1}$, Vidyut Bhatia ${ }^{2}$, Leena Mendiratta ${ }^{1}$
}

\section{Hena Butta ${ }^{1 *}$, Akshay \\ Kapoor ${ }^{2}$, Anupam Sibal' Raman Sardana ${ }^{1}$, Vidyut Bhatia $^{2}$, Leena Mendiratta ${ }^{1}$}

\author{
'Department of Microbiology, \\ Indraprastha Apollo Hospitals, \\ New Delhi, INDIA. \\ ${ }^{2}$ Department of Pediatric \\ Gastroenterology and Hepatology, \\ Indraprastha Apollo Hospitals, \\ New Delhi, INDIA.

\section{Correspondence} \\ Dr Hena Butta \\ DNB (Microbiology), Consultant \\ Microbiology, Department of \\ Microbiology, Indraprastha Apollo \\ Hospitals, Sarita Vihar, New Delhi-110076, \\ INDIA. \\ Mobile no: 91-8447233605 \\ Email: drhena_b@apollohospitalsdelhi.com \\ History \\ - Submission Date: 07-02-2017 \\ - Revised Date: 10-11-2017 \\ - Accepted Date: 14-12-2017
}

DOI : 10.5530/ijmedph.2018.1.2

Article Available online

http://www.ijmedph.org/v8/i1

\section{Copyright}

(C) 2018 Phcog.Net. This is an openaccess article distributed under the terms of the Creative Commons Attribution 4.0 International license.

\begin{abstract}
Purpose: Ulcerative colitis (UC) is a chronic idiopathic inflammatory bowel disease of intestines. The high cost of second line drugs along with the need for lifelong therapy are the most distressing factors demanding innovative therapy for treating this disease. Fecal microbial transplantation (FMT) can be used as an adjunct for treating UC. We performed FMT in a six year old male child who was being treated for ulcerative colitis with conventional therapy for the last two years with variable response to therapy. This therapy also has good potential as salvage therapy for refractory multidrug resistant Gram negative organisms and Clostridium difficile infection. Methods: After taking appropriate informed consent, a healthy adult donor from the family was thoroughly screened for various infections (as per protocol). Donor fecal preparation was done taking strict aseptic precautions and FMT was given as retention enema for $1 \mathrm{~h}(60 \mathrm{ml}$ enema every $15 \mathrm{~min}$ ) daily for 5 days. Patient was monitored for adverse event (as per protocol). Results: The patient tolerated the enema well. The Paediatric Ulcerative Colitis Activity Index (PUCAI) reduced from 35 to 15 after completion of five days of therapy. There has been no relapse since FMT and the child continues to be asymptomatic with a stable low PUCAI score 6 months post therapy. Conclusion: Our study is a step and encouragement towards the usage of FMT for pediatric patients with ulcerative colitis showing variable response to conventional treatment. However, more future studies are required to authenticate this treatment modality.
\end{abstract}

Key words: Enema, Fecal Microbial Transplantation (FMT), PUCAI score, Ulcerative colitis.

\section{INTRODUCTION}

Ulcerative colitis (UC) is a chronic idiopathic inflammatory bowel disease (IBD) of intestines in genetically predisposed individuals associated with well-defined extra-intestinal complications. The disease course is characterized by spontaneous remission and relapses. The pathophysiology involves an aberrant immune response to the gut microbiota. ${ }^{1}$ Incidence rates of IBD are increasing in areas of the world previously unaffected by the disease. In the first half of twentieth century the annual incidence for ulcerative colitis was reported to be $6.0 / 100,000$ for the period 19341944 and 1.9/100,000 for the period 1935-1954 from Rochester Minnesota. ${ }^{2,3}$ Also, Jewish ethnicity and high socioeconomic status was found to be a risk factor for the development of IBD. ${ }^{4}$ At the end of the $20^{\text {th }}$ century an epidemic of IBD was noticed mainly affecting Western Europe and Northern America. In the twenty-first century, the increase in incidence of IBD was reported from various regions of the world including Asia, Africa, Middle East and an increase in trend was reported from other areas of Europe and America. ${ }^{5}$ Ulcerative colitis was first reported from India in the late 1930 s. ${ }^{6}$ Later on the existence of the disease was reported in the northern and western parts of the country. Amongst Asian countries, India has the highest incidence and prevalence of ulcerative colitis followed by Japan and Korea. ${ }^{6}$ With the changing epidemiology of IBD, it is predicted that there will be an increase in IBD patients in Asia, Southern America as well as East Europe. ${ }^{5}$ The high cost of second line drugs along with the need for life long therapy are the most distressing factors demanding the new innovative therapy for the treatment of the disease. Garg et al have evaluated the efficacy and safety of helminth treatment for induction of remission in inflammatory bowel disease. ${ }^{7}$ However, currently, there is insufficient evidence to allow any firm conclusions regarding this unconventional modality of treatment and this warrants further studies. Hence, there is need for diverse and innovative strategies to find out the etiology, prevention and treatment of this disease.

Fecal microbial transplantation (FMT) is a new hope for the treatment of ulcerative colitis patients. ${ }^{8}$ It involves the delivery of stool from a healthy prescreened donor to an individual with disease. FMT is being recognized as a treatment modality for the various gastrointestinal diseases associated with intestinal dysbiosis. The first use of FMT in humans was reported in 1958 in a case series of 4 patients with pseudomembranous enterocolitis. ${ }^{9}$ There are various studies showing the usefulness of FMT for the treatment of recurrent Clostridium difficile infections (CDI). ${ }^{10,11,12}$ FMT restores the healthy commensal bacteria in the intestines thereby correcting intestinal
Cite this article : Butta H, Kapoor A, Sibal A, Sardana R, Bhatia V, Mendiratta L. Fecal Microbial Transplant- A New Hope of Treatment for Ulcerative Colitis. Int J Med Public Health. 2018;8(1):7-10. 
dysbiosis associated with Clostridium difficile infection. The exact etiology of ulcerative colitis is unknown. The etiologic factors potentially found to be contributing to ulcerative colitis include genetic factors, immune system reactions, environmental factors, nonsteroidal anti-inflammatory drug (NSAID) use, low levels of antioxidants, psychological stress factors, a smoking history, and consumption of milk products. The environmental factor playing a major role in the disease etiology is the altered bacterial flora. ${ }^{13}$ So, the restoration of bacterial flora can be an approach for the treatment of patients with ulcerative colitis. There are certain safety and ethical issues associated with FMT. The issues associated with FMT are adequate screening of donor fecal matter, cleanliness and worsening of ulcerative colitis. FMT may be administered to the proximal colon via colonoscope, the distal lower gastrointestinal tract via enema, rectal catheter, and the upper GI tract through a nasogastric tube, a nasoduodenal tube or a gastroscope. ${ }^{14}$

We are hereby reporting our findings in a child with ulcerative colitis who was treated with fecal microbial transplantation at our institute. To the best of our knowledge, the use of FMT for the treatment of ulcerative colitis is being reported for the first time in India.

\section{MATERIAL AND METHODS}

\section{Case Selection}

This six year old child was diagnosed as case of ulcerative colitis on the basis of history, examination, serology, colonoscopy and histopathology. He was initially admitted at our institute in February 2012 with history of loose, watery stools with mucus and blood for one year associated with periumbilical pain. Repeated microscopic examination of stool sample showed 20-25 pus cells per high power field and visible blood. No cyst or ova of parasites were detected in any of the stool samples. Special stains for parasites also did not reveal any abnormal findings. The culture examination of stool showed no growth of enteropathogenic bacteria. Testing for Clostridium difficile toxin was negative on three separate stool specimens. He was given trial of antimicrobials like cefixime, metronidazole and ofloxacin with no response. He underwent colonoscopy which was suggestive of pancolitis. Laboratory investigations including complete blood count, liver enzymes, albumin, erythrocyte sedimentation rate (ESR) and C-reactive protein (CRP) were within normal limits. Other investigations also did not reveal any immunodeficiency disorder. Prednisolone and Mesalamine was started. He improved significantly after this but relapsed on tapering steroids. In April 2013, patient was started on specific carbohydrate diet (no dairy, no grains and restricted carbohydrate) in addition to conventional therapy to which he responded favourably and remained symptom free till October 2013. He subsequently had two more relapses on tapering steroids. $\mathrm{He}$ was again started on steroids, 5-ASA derivatives and specific carbohydrate diet. The response to treatment was intermittent with relapses interspersed between periods of symptomatic remission. In view of inadequate response to treatment and parents opting against the use of immunosuppressive they contacted our institute for FMT. The disease severity of the child was ascertained by Paediatric Ulcerative Colitis Activity Index(PUCAI). ${ }^{15}$ The various components of PUCAI with their grading are abdominal pain $(0,5,10)$, rectal bleeding $(0,10,20,30)$, stool consistency of most stools $(0,5,10)$, number of stools per $24 \mathrm{~h}(0,5,10,15)$, nocturnal stools (any episode causing wakening- 0,10$)$ and activity level $(0,5,10)$.The sum of each of the component is done to calculate PUCAI (Total sum 0-85). On the basis of PUCAI, the disease severity in our patient was defined as moderate $(\mathrm{PUCAI}=35)$. Hence, the procedure for FMT was planned in June 2014.

\section{Donor selection and screening tests}

In our case, the parents of the patient approached us for FMT procedure. Institutional ethics committee clearance was obtained prior to the procedure. Appropriate consent form was signed by the parents stating the investigational nature of the FMT treatment, its possible side-effects and the procedure being conducted for the first time by our hospital.

Apparently healthy, immunocompetent adult donor from the family (patient's biological father) with no previous history of C.difficile infection/ inflammatory bowel disease was screened for various infections. The other exclusion criteria for the donor were any history of Irritable Bowel Syndrome (IBS), abdominal pain, gastrointestinal malignancy, prolonged diarrhea or blood in stool, antibiotic/antimicrobial use within one month before FMT. The donor was screened for Hepatitis A, B and C (Anti HAV IgM, HBsAg, AntiHBs, AntiHBc, Anti HCV), Epstein Barr Virus (viral capsid antigen IgM), Cytomegalovirus (AntiCMVIgM), Syphilis (VDRL and TPHA), HIV (Anti HIV 1 and 2). The various stool tests performed were routine and microscopic examination for ova and cyst, occult blood, Modified Zeihl Neelsen staining for Cryptosporidium, stool culture for Salmonella, Shigella, Vibrio, diarrheagenic Escherichia coli, Listeria, Campylobacter, Real Time PCR for Yersinia, Campylobacter, Clostridium difficile, Salmonella, Shigella/Enteroinvasive Escherichia coli, Enterohaemorrhagic/verotoxin producing Escherichia coli and Clostridium difficile toxin detection. All the tests done on the donor were found to be satisfactory.

\section{Donor fecal sample preparation}

The donor was counselled properly regarding sample collection. The donor had to produce about $100 \mathrm{~g}$ of fecal specimen daily for five consecutive days. The sample preparation was done in biosafety cabinet in a designated laboratory which was properly cleaned and disinfected with three cycles of ecoshield. Fecal sample was blended with $250 \mathrm{ml}$ sterile warm $\left(37.8^{\circ} \mathrm{C}\right)$ normal saline for one min. This blended mixture was filtered through two sterile gauze pieces to remove larger sediments. Filtered fecal preparation was aliquoted in presterilized empty enema pouches of $60 \mathrm{ml}$ each and these enema pouches were kept in warm water bath $\left(37.8^{\circ} \mathrm{C}\right)$ until FMT was administered. FMT was administered within $6 \mathrm{~h}$ of production of stool sample.

\section{FMT procedure}

No bowel preparation (clean out or antibiotic pre-treatment) was done before FMT. Patient received FMT as retention enema for $1 \mathrm{~h}(60 \mathrm{ml}$ enema every $15 \mathrm{~min}$ ) daily for 5 days. Each $60 \mathrm{ml}$ enema was infused slowly over a time period of $5 \mathrm{~min}$. The patient was then turned 180 degrees from left lateral to right lateral position and then back to left lateral position slowly during a 10 min period. Patient was monitored for $30 \mathrm{~min}$ after FMT for any immediate adverse events and discharged.

\section{RESULTS}

Our patient tolerated the enema well. There was no immediate leaking of enema during administration and the patient was able to retain enema for at least one $h$ after administration. On the first day, only three enemas could be given because the child was apprehensive. Enema was given under sedation for next four days after taking informed consent from the patient attendants. The Paediatric Ulcerative Colitis Activity Index (PUCAI) at the time of FMT was 35 and it was reduced to 15 after five days of completion of treatment. Continuous follow-up of the child is being done. Presently, the child is on normal diet and his medication is being tapered. The child is showing improvement in symptoms and has not had any relapse since then. 


\section{DISCUSSION}

In case of recurrent Clostridium difficile infections, FMT has demonstrated a rapid response and cure rate exceeding $90 \%$ with a negligible significant adverse event rate, regardless of route. ${ }^{16}$ The published studies showing benefits of FMT are mainly limited for recurrent Clostridium difficile infections. There are not much published studies showing benefits of FMT for the treatment of ulcerative colitis. Angelberger S et al., in their study on temporal bacterial community dynamics among ulcerative colitis patients post FMT found clinical response in one out of five patients with moderate to severe ulcerative colitis. ${ }^{17}$ Kunde $S$ et al., in their study on FMT on ten children, 7 to 21 years of age, with mild-tomoderate ulcerative colitis found fecal enemas to be efficacious, feasible and tolerated by children. They reported $78 \%$ clinical response within 1 week; clinical remission in $33 \%$ of patients; and only mild and selflimiting adverse events. ${ }^{18}$ Dr. Paul Moayyedi and his colleagues from McMaster University in Hamilton, Ontario, Canada evaluated ambulatory patients with active ulcerative colitis with FMT and presented results for the first 61 of an anticipated 130 patients to complete the trial at Digestive Disease Week 2014. They did not found significant improvement in patients with ulcerative colitis treated with fecal microbiota therapy after 6 weeks of treatment. Patients who received FMT reported some improvement after treatment, but they were not in remission at 7 weeks. However, in a later study conducted on 70 patients, Moayyedi et al observed that FMT induces remission in a significantly greater percentage of patients with active ulcerative colitis than placebo, with no difference in adverse events. ${ }^{19}$ Rossen et al in their study to assess the efficacy and safety of FMT for patients with ulcerative colitis, observed that there was no statistically significant difference in clinical and endoscopic remission between patients with ulcerative colitis who received fecal transplants from healthy donors and those who received their own fecal microbiota. However, the microbiota of responders had distinct features from that of non-responders. ${ }^{20}$

In India, Ulcerative colitis is now being diagnosed more frequently. In North India, it has been found to be responsible for a hospital admission rate of $12 / 10,000 .{ }^{21}$ The proportion of IBD have been reported to be $0.03 \%$ per year $(33$ per 100,000$)$ of all new pediatric patients attending a South Indian hospital with pediatric patients accounting for approximately $7 \%$ of all new cases of IBD seen annually. ${ }^{22}$ Environmental factors such as diet and urbanization play an important role in the development of ulcerative colitis. So, the disease is being increasingly reported in parts of world where these diseases used to be rare. The goals of IBD treatment are to induce and maintain remission of both clinical symptoms and mucosal inflammation, and to re-establish the intestinal barrier, so as to reduce relapse and complication, and to improve quality of life. In cases of failure of remission or maintenance, new biological or surgery should be considered. ${ }^{23}$ FMT can be considered for such cases. There is no consensus on the optimal protocol of FMT but the overall success rate of FMT has been reported to be $90 \%$ irrespective of the methodology used. We have used the retention enema technique in our case and it was found to be feasible and acceptable.

FMT has successfully been used for the decolonization of the gut of a young girl who persistently harboured multidrug-resistant Klebsiella pneumoniae bacteria causing recurrent infections. Post FMT carbapenemresistant Klebsiella was not detectable in a stool sample and remained undetectable in 4 stools over the next 8 months and also there was no further clinical evidence of bacterial infection in the patient. ${ }^{24}$

\section{CONCLUSION}

Our study is a step towards the usage of FMT for patient with ulcerative colitis and is an encouraging step for the future use of this therapy for the patients with poorly controlled ulcerative colitis. Further studies are required for the use of FMT in children with ulcerative colitis and also to establish its emerging role in replacement of gut flora as a therapy for managing chronic multidrug resistant organisms in the patients.

\section{CONFLICT OF INTEREST}

The authors declare no conflict of interest.

\section{ABBREVIATIONS USED}

Anti CMV: Antibodies against Cytomegalovirus; Anti HAV: Antibodies against Hepatitis A Virus; Anti HBc: Antibodies against Hepatitis B core antigen; Anti HBs: Antibodies against Hepatitis B surface antigen; Anti HCV: Antibodies against Hepatitis C Virus; 5-ASA: 5-Aminosalicylic acid; C: Celsius; g: gram; h: hour; HBsAg: Hepatitis B surface antigen; HIV: Human Immunodeficiency Virus; IgM: Immunoglobulin M; ml: milliliter; min: minute; PCR: Polymerase Chain Reaction; VDRL: Venereal Disease Research Institute; TPHA: Treponema Pallidum Haemagglutination.

\section{REFERENCES}

1. Loftus EV. Clinical epidemiology of inflammatory bowel disease: Incidence, prevalence and environmental influences. Gastroenterology. 2004;126(6):1504-17.

2. Stonnington CM, Philips SF, Melton III LJ, Zinsmeister AR. Chronic ulcerative colitis: incidence and prevalence in a community. Gut. 1987;28(4):402-9.

3. Sedlack RE, Nobrega FT, Kurland LT, Sauer WG. Inflammatory colon disease in Rochester, Minnesota, 1935-1964. Gastroenterology. 1972;62(5):935-41.

4. Acheson ED. The distribution of ulcerative colitis and regional enteritis in United States veterans with particular reference to Jewish religion. Gut. 1960;1(4):291-3.

5. Ekbom A. The changing Epidemiology of IBD. In: Inflammatory Bowel Disease Diagnosis and Therapeutics. Clinical Gastroenterology. $2^{\text {nd }}$ ed. Cohen RD, editors. Humana Press, NewYork. 2011:17-26.

6. Puri AS. Epidemiology of ulcerative colitis in South Asia. Intest Res. 2013; $11(4): 250-5$

7. Garg SK, Croft AM, Bager P. Helminth therapy (worms) for induction of remission in inflammatory bowel disease. Cochrane Database Syst Rev. 2014;(1):CD009400

8. Kahn SA, Vachon A, Rodriquez D, Goeppinger S, Surma B, Marks J, et al . Patient Perceptions of FecalMicrobiota Transplantation for Ulcerative Colitis. Inflamm Bowel Dis. 2013;19(7):1506-13.

9. Eiseman B, Silen W, Bascom GS, Kauvar AJ. Fecal enema as an adjunct in the treatment of pseudomembranous enterocolitis. Surgery. 1958;44(5):854-9.

10. Yoon SS, Brandt LJ. Treatment of refractory/recurrent $C$. difficile-associated disease by donated stool transplanted via colonoscopy: a case series of 12 patients. J Clin Gastroenterol. 2010;44(8):562-6.

11. Bakken JS. Fecal bacteriotherapy for recurrent Clostridium difficile infection. Anaerobe. 2009;15(6):285-9.

12. Mattila E, Uusitalo-Seppala R, Wuorela M, Lehtola L, Nurmi H, Ristikankare M et al. Facal transplantation, through colonoscopy, is effective therapy for recurrent Clostridium difficile infection. Gastroenterology. 2012;142(3):490-6.

13. Almeida MG, Kiss DR, Zilberstein B, Quintanilha AG, Teixeira MG, Habr-Gama A. Intestinal mucosa-associated microflora in ulcerative colitis patients before and after restorative proctocolectomy with an ileoanal pouch. Dis Colon Rectum. 2008;51(7):1113-9

14. Vaishnavi C. Fecalmicrobiota transplantation for management of Clostridium difficile infection. Indian J Gastroenterol. 2014;33(4):301-7.

15. Turner D, Travis SPL, Griffiths AM, Ruemmele FM, Levine A, Benchimol El, et al. Consensus for managing acute severe ulcerative colitis in children: A systematic review and joint statement from ECCO, ESPGHAN, and the Porto IBD working group of ESPGHAN. Am J Gastroenterol. 2011;106(4):574-88.

16. Rubin TA, Gessert CE, Aas J, Bakken JS. Fecal microbiome transplantation for recurrent Clostridium difficile infection: Report on a case series. Anaerobe. 2013;19:22-6.

17. Angelberger S, Reinisch W, Makristathis A, Lichtenberger C, Dejaco C, Papay $P$, et al. Temporal bacterial community dynamics vary among ulcerative colitis patients after fecal microbiota transplantation. Am J Gastroenterol. 2013;108(10):1620-30

18. Kunde S, Pham A, Bonczyk S, Crumb T, Duba M, Conrad H, et al . Safety, tolerability, and clinical response after fecal transplantation in children and young adults with ulcerative colitis. J Pediatr Gastroenterol Nutr. 2013;56(6):597-601.

19. Moayyedi P, Surette MG, Kim PT, Libertucci J, Wolfe M, Onischi C, et al . Fecal Microbiota Transplantation induces remission in patients with active ulcerative colitis in a randomized controlled trial. Gastroenterology. 2015;149(1):102-9. 
20. Rossen NG, Fuentes S, van der Spek MJ, Tijssen JG, Hartman JH, Duflou A, et al . Findings From a Randomized Controlled Trial of Fecal Transplantation for Patients With Ulcerative Colitis. Gastroenterology. 2015;149(1):110-8.

21. Sood A, Midha V, Sood N, Puri S, Kaushal V. Profile of ulcerative colitis in a North Indian Hospital. J Indian Acad Clin Med. 2000;5:125-9.

22. Avinash B, Dutta AK, Chacko A. Pediatric Inflammatory Bowel Disease in South India. Indian Pediatr. 2009;46:639-40.
23. Ouyang Q, Tandon R, Goh KL, Pan GZ, Fock KM, Fiocchi C, et al . Management consensus of Inflammatory Bowel Disease for the Asia-Pacific region. J Gastroenterol Hepatol. 2006;21(12):1772-82.

24. Medscape Medical News. Fecal transplant eliminates persistent MDR infection. Available at: http://www.medscape.com/viewarticle/833502 (Accessed October 20, 2014).

Cite this article : Butta H, Kapoor A, Sibal A, Sardana R, Bhatia V, Mendiratta L. Fecal Microbial Transplant- A New Hope of Treatment for Ulcerative Colitis. Int J Med Public Health. 2018;8(1):7-10. 